\title{
Stochastic Filter methods for generally Constrained Global Optimization
}

\author{
C.J. Price • M. Reale • B.L. Robertson
}

the date of receipt and acceptance should be inserted later

The final publication is available at link.springer.com

Abstract A filter based template for bound and otherwise constrained global optimization of non-smooth black-box functions is presented. The constraints must include finite upper and lower bounds, and can include nonlinear equality and inequality constraints. Almost sure convergence is shown for a wide class of algorithms conforming to this template. An existing method for bound constrained global optimization (OSCARS) is easily modified to conform to this template. Numerical results show the modified OSCARS is competitive with other methods on test problems including those listed by Koziel and Michalewicz.

keywords: filter, ARS, OSCARS, direct search, bound and otherwise constrained global optimization.

\section{Introduction}

The bound and otherwise constrained global optimization problem is

$$
\min _{x \in \Omega} f(x) \quad \text { subject to } \quad g(x) \leq 0
$$

where $f$ is the objective function. The constraint function $g$ is a vector function mapping $\mathbb{R}^{n} \rightarrow \mathbb{R}^{q}$, and $\Omega \subset \mathbb{R}^{n}$ is a finite box of the form

$$
\Omega=\left\{x \in \mathbb{R}^{n}: L_{i} \leq x_{i} \leq U_{i} \quad \forall i=1,2, \ldots, n\right\} .
$$

Here $x_{i}$ is the $i^{\text {th }}$ element of $x$, and all upper and lower bounds $U_{i}$ and $L_{i}$ are finite, with $L_{i}<U_{i}$ for all $i$. For simplicity only general inequality constraints are included in (1). Equality constraints can be included as a pair of inequality constraints, without loss of generality.

C.J. Price · M. Reale · B.L. Robertson Mathematics and Statistics, University of Canterbury, Christchurch, New Zealand, E-mail: C.Price@math.canterbury.ac.nz, marco.reale@canterbury.ac.nz, blair.robertson@canterbury.ac.nz 
We assume that $f$ and $g$ are black-box functions which, when supplied with a point $x$, return $f(x)$ and $g(x)$. It is also assumed that $f$ and $[g]_{+}$are Lebesgue measurable, where $[g(x)]_{+} \in \mathbb{R}^{q}$ takes the element-wise maximum of $g(x)$ and zero. Finally we assume $f$ is bounded below, and that $f$ and $[g]_{+}$are lower semicontinuous at points of interest.

There are a number of different strategies to address (1), some of which reduce (1) to minimizing an appropriate penalty function over $\Omega$ [5]. A related approach is [9], which applies the DIRECT method of Jones et al. [10] to an auxiliary function. Interval methods $[13,20]$ have also been used to solve (1) via a branch and bound strategy. Other approaches include the use of approximations to the objective function [17], and filled functions [23]. In addition there are many stochastic methods including fish swarm [16], particle swarm [21], simulated annealing [8], evolutionary algorithms [19], and electromagnetism-like methods [1].

Many of the methods above make use of information not available in nonsmooth black-box optimization, such as differentiability of $f$ and $g$ [5,22-24], or the functions' analytic forms [13,20]. In contrast methods such as $[8,9,16,19,21]$ require only the functions' values at each iterate. Since these methods neither use nor require additional information or properties about $f$ and $g$, they can be easily applied to non-smooth black-box functions, as is the intent of this paper. Allowing non-linear equality constraints means feasible point algorithms are not an option. Dealing with infeasible points presents two conflicting aims: attaining feasibility and minimizing $f$. One way to reconcile these two aims is to use an exact penalty function, which requires an appropriate choice of penalty parameter(s). Di Pillo et al. [5] examine this when $f$ and $g$ are continuously differentiable. Since (1) might not be smooth, picking such parameters is usually hard. One could work with a variety of different penalty parameter values simultaneously, but, depending on the relative scaling of $f$ and $g$, the best such values could be arbitrarily large or small. Instead, like [8], we opt for a filter.

In general terms, global optimization algorithms calculate $f$ and $g$ at a sequence of sample points in $\Omega$. Subsequent sample points are selected based on information from earlier sample points. For example, a method could explore more thoroughly the vicinity of promising sample points than other parts of $\Omega$. One way to identify promising sample points is by their objective function values and constraint violations. If we retain only points which are clearly better than any other sample point in either objective function value or constraint violation or both, then the points we retain form a filter. Discarding poor sample points in this fashion can lower computational overheads and storage requirements as well as improve sampling effectiveness.

In this paper we formulate a general framework for solving (1) using filters [6]. The basic approach of the filter template is to generate an infinite sequence of iterates $\left\{x^{(k)}\right\}_{k=0}^{\infty}$ in $\Omega$. An infinite subset of these iterates are drawn randomly from $\Omega$ in order to guarantee convergence almost surely. Others are drawn from subregions of $\Omega$ with the intent of improving the convergence rate. The existence of a sequence of filter points converging to solution(s) of (1) with probability one can be shown under mild conditions. A modified version of one side cut accelerated random search (hereafter OSCARS) [14] conforming to the filter template is also tested.

Accelerated random search (ARs) [3], and its offshoot oscARs [14], are methods for bound constrained global optimization which use the objective function only 
to determine which of a pair of points is better than the other. Hence they can easily be modified to address other problems such as (1) when an alternative way of doing pairwise comparisons (e.g. via filters) exists.

ARs searches about the current best known point (hereafter the control point) by polling randomly in a finite sequence of nested boxes until a better point is found. This better point then becomes the next control point, and the process is repeated. If the sequence of nested boxes is exhausted without improvement, ARS continues polling cyclically through the sequence of boxes. The first box in the sequence is $\Omega$. Each subsequent box is contained in its predecessor, and is the intersection of a scaled version of $\Omega$ centred on the current iterate with $\Omega$ itself.

The principal difference between ARS and OSCARS is the latter does not shrink the polling box in every direction after a failed poll. Using $c$ as the control point, and $x^{(k)}$ as the $k^{\text {th }}$ random polling point, OscARs shortens the box along one coordinate axis so that $c$ remains in the shortened box, but $x^{(k)}$ does not. The axis used is the one along which $x^{(k)}$ and $c$ have the greatest separation. Like ARs, this process continues until either a better point than $c$ is found, or the polling box becomes too small, after which the polling box is reset to $\Omega$. If a better point has been found, then $c$ is set to this better point.

This paper is organized as follows. The next section formally defines what is meant by a solution to (1). Section 3 describes the stochastic filter template, and establishes convergence almost surely. Sections 4 and 5 describe and numerically test a filter version of OSCARS which conforms to the template. Concluding remarks appear in section 6 .

\section{Theoretical Development}

The feasible region $\Phi$ is

$$
\Phi=\{x \in \Omega: g(x) \leq 0\} .
$$

The total constraint violation at a point $x \in \Omega$ is measured using

$$
\theta(x)=G\left(\left\|[g(x)]_{+}\right\|\right)
$$

where $[g(x)]_{+} \in \mathbb{R}^{q}$ takes the element-wise maximum of $g(x)$ and zero, and $G$ is a continuous strictly increasing function satisfying $G(0)=0$. The inclusion of the function $G$ allows such choices as

$$
\theta(x)=\left\|[g(x)]_{+}\right\|_{2}+\left\|[g(x)]_{+}\right\|_{2}^{2}
$$

which is used to generate the numerical results presented herein. Continuity of $G$ ensures points with small values for $[g]_{+}$have correspondingly small values for $\theta$. The use of weighted norms in (2) permits constraint scaling to be included.

Our approach is intended to cope with 'black-box' objective and constraint functions. The convergence analysis requires that the objective function $f$ be Lebesgue measurable, bounded below, and lower semi-continuous at points of interest. The minimum conditions on $g$ are that $\theta$ is both Lebesgue measurable, and lower semi-continuous. This implies the feasible region $\Phi$ is a closed set under the standard topology on $\mathbb{R}^{n}$ restricted to $\Omega$. This topology is used exclusively in this paper. 
First we define precisely what is regarded as an acceptable solution to (1). This definition makes use of infeasible points, and so we define the set of points $\Phi(\eta)$ in which the constraint violation is at most $\eta$ as follows.

$$
\Phi(\eta)=\{x \in \Omega: \theta(x) \leq \eta\} .
$$

Lower semi-continuity of the measure of infeasibility $\theta$ means each $\Phi(\eta)$ is a closed set. Since $\Omega$ is bounded, each $\Phi(\eta)$ is compact. In particular, the feasible region $\Phi=\Phi(0)$ is compact.

Our intent is to explore methods which solve (1) by random search over $\Omega$ and various subregions of $\Omega$. Convergence is guaranteed almost surely through sample points (or iterates) chosen randomly from $\Omega$. Hence sets of Lebesgue measure zero are essentially invisible because sampling them is a probability zero event. This motivates the following definitions. Let $m_{L}$ be the Lebesgue measure.

Definition 1 Essential global minimum of $f$ over $\Phi(\eta)$ : Let $\gamma(\eta)$ be the infimum of infinity and all values $f_{\text {test }}$ for which

$$
m_{L}\left(\left\{z \in \Phi(\eta): f(z)<f_{\text {test }}\right\}\right)>0 .
$$

Then $\gamma(\eta)$ is the essential global minimum of $f$ over $\Phi(\eta)$. Any point $x \in \Phi(\eta)$ satisfying $f(x) \leq \gamma(\eta)$ is called an essential global minimizer of $f$ over $\Phi(\eta)$.

When $\eta=0, \gamma(0)$ gives the essential global minimum of $f$ over the feasible region $\Phi$. The definition of $\gamma(0)$ only considers feasible points. Allowing nearly feasible points to be considered gives:

Definition 2 Essential global solution of (1): Let $f_{\mathrm{egs}}^{*}$ be the infimum of infinity and all values $f_{\text {test }}$ which satisfy

$$
m_{L}\left(\left\{z \in \Phi(\epsilon): f(z)<f_{\text {test }}\right\}\right)>0, \quad \forall \epsilon>0 .
$$

Then $f_{\mathrm{egs}}^{*}$ is the essential global solution value of (1). Any feasible point $x$ satisfying $f(x) \leq f_{\text {egs }}^{*}$ is called an essential global solution point of (1).

The set of all essential global solution points is denoted $S^{*}$. Given that we are solving (1) by generating random sample points in $\Omega$ and various subregions of $\Omega$, attaining the essential global solution value is the best we can realistically aim for.

Clearly $\gamma(\eta) \leq f_{\text {egs }}^{*}$ for all positive $\eta$. This follows directly from replacing " $\forall \epsilon>$ 0 " with " $\epsilon=\eta$ " in (5). In fact

$$
\sup \{\gamma(\eta): \eta>0\}=\lim _{\eta \downarrow 0} \gamma(\eta)=f_{\text {egs }}^{*}
$$

where the limit exists and equals the supremum because $\gamma$ is a decreasing function of $\eta$. Clearly $f_{\text {egs }}^{*}$ is an upper bound on this limit. Now, for any $f_{\text {test }}, f_{\text {test }}$ satisfies (5) iff $f_{\text {test }}$ satisfies (4) for all $\eta>0$. The right hand equality in (6) follows because $f_{\text {egs }}^{*}$ is the infimum of all $f_{\text {test }}$ values satisfying (5). Note that putting $\eta=0$ in $\gamma(\eta)$ does not yield $f_{\text {egs }}^{*}$. The latter's definition considers points which do not lie in $\Phi$, whereas the former (with $\eta=0$ ) does not. The difference between these quantities is easily seen from the following example. 


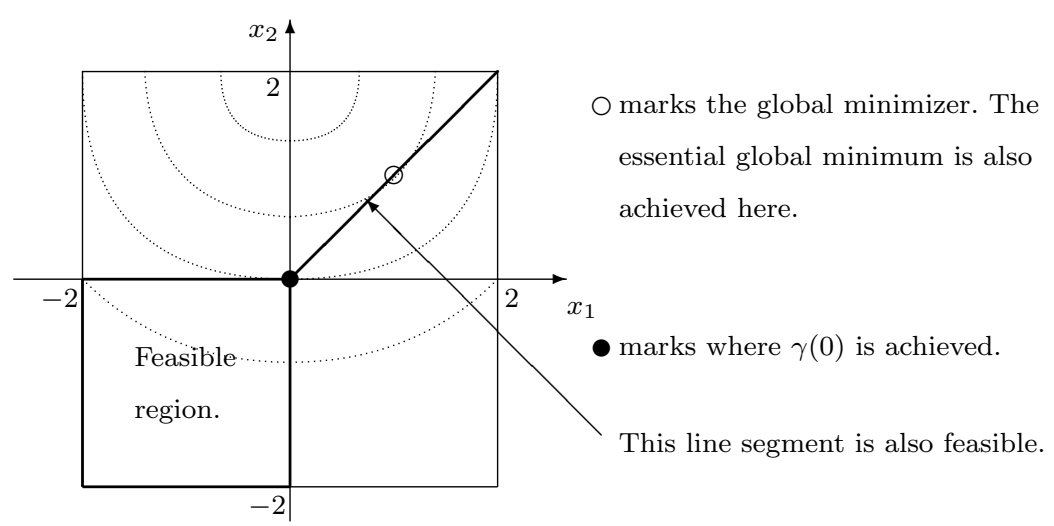

Fig. 1 The feasible region (boundary in bold) and level curves of $f$ (dotted) for Example 1.

\section{Example 1:}

$$
f=x_{1}^{2}+\left(x_{2}-2\right)^{2} \quad g=\left(x_{1}-x_{2}\right)^{2} \max \left(x_{1}, x_{2}\right), \quad \text { and } \quad \Omega=[-2,2]^{2} .
$$

The feasible region consists of the union of bottom left quadrant of $\Omega$ and the line $x_{1}=x_{2}$ with $0 \leq x_{1} \leq 2$. The global minimum and essential global solution $f_{\text {egs }}^{*}$ both equal 2, and occur at $(1,1)$, as is shown in Figure 1 . In contrast $\gamma(0)=4$ and this value is achieved at the origin. If $\Omega$ is altered to $\Omega=[0,2]^{2}$ then the global minimizer and essential global solution are unchanged, but $\gamma(0)=\infty$.

\section{Filters and Convergence}

The notion of a filter [6] is based on a two dimensional 'ordering' of points in $\Omega$ in terms of their $f$ and $\theta$ values. If one point ( $x$ say) has lower values for both $f$ and $\theta$ than another (say $y$ ), then $x$ is clearly a better point than $y$. Similarly $x$ is clearly worse if both its values are higher; and $x$ is comparable to $y$ if one value is higher, and the other lower. At highly infeasible points objective function values are often uninformative, and $f$ might not even be defined in such regions. For such points one point is better than another only if it has lower infeasibility. To be precise, a point $x$ weakly dominates a second point $y$ (written $x \preceq y$ ) if and only if either

(a) both $f(x) \leq f(y)$ and $\theta(x) \leq \theta(y)$; or

(b) both $\theta(y)>\Theta$ and $\theta(x) \leq \theta(y)$.

Here $\Theta$ is the $\theta$ value above which points which are regarded as highly infeasible. If either both inequalities in (a) hold and at least one is strict, or both inequalities in (b) hold and the second one is strict, then we say $x$ dominates $y$ (written $x \prec y$ ).

A filter $F$ is simply a set of sample points with the property that no point in the filter is dominated by any other point in the filter [6]. In contrast to [6], feasible points are included directly in our filter, and do not have any special status there. A filter can be used to divide $\Omega$ into three subsets according to the values taken by $f$ and $\theta$. The first of these is the set of 'worse' points. This set contains all points in $\Omega$ which are dominated by at least one point in the filter. The second 
set contains all points which dominate at least one filter point. This is the set of 'better' points. All remaining points neither dominate, nor are dominated by, any point in the filter $F$. These points are called comparable points to the filter $F$.

Filters may be used to solve (1) when the sequence of iterates $\left\{x^{(k)}\right\}_{k=0}^{\infty}$ contains an infinite subsequence of samples drawn randomly from $\Omega$. At iteration $k$ the filter $F_{k} \subseteq\left\{x^{(0)}, \ldots, x^{(k)}\right\}$ is formed by updating the previous iteration's filter with the current iterate $x^{(k)}$. If $x^{(k)} \prec z$ for some $z \in F_{k-1}$ then $F_{k}$ is set equal to $F_{k-1} \cup\left\{x^{(k)}\right\}$. All points in $F_{k}$ which are dominated by $x^{(k)}$ are then removed from $F_{k}$. Alternatively if $z \prec x^{(k)}$ for some $z \in F_{k-1}$, then $F_{k}$ is set equal to $F_{k-1}$. The remaining case is when $x^{(k)}$ is comparable to $F_{k-1}$. Following [6] we include all comparable points in the filter.

The tactic of including all unfiltered (i.e. comparable and better points) in the filter can lead to the size $\left|F_{k}\right|$ of $F_{k}$ increasing steadily as $k \rightarrow \infty$. Whilst this is not desirable, it is not uncommon for global optimization methods to have storage requirements which increase steadily as $k$ increases. For example $[2,10,15,18]$ store and process every iterate generated, or at least a fixed percentage of them. Clearly filters usually discard many iterates, and so this approach is not unreasonable. Nevertheless strategies which limit the number of points $|F|$ in $F$ might be useful. We examine one such pruning process, and then describe the filter template.

When the size $|F|$ of $F$ becomes excessive, the filter points are grouped into bands, where points in the same band have similar $\theta$ values. Pruning retains only the points which have the least $f$ value in some band, with one exception. The point which is the least infeasible infeasible point $w$ in $F$ is also retained, even when $F$ contains a feasible point. Without automatically retaining $w$ there is a risk of failing to reduce infeasibility by cyclically generating points such as $w$ with new low values of $\theta$, and then deleting them during pruning because of another filter point $z$ in the same band with $f(z)<f(w)$ and $\theta(z)>\theta(w)$.

A set of pruning marks $\mathcal{M} \subseteq[0, \infty)$ is chosen, where

$$
0 \in \mathcal{M} \text { and } \inf \{\mu \in \mathcal{M}: \mu>0\}=0 .
$$

A filter $F$ can be pruned using these marks to get a filter $P$ as follows. The least infeasible infeasible point is always included in $P$. The remaining points in $P$ are those which minimize $f$ over $F \cap \Phi(\mu)$ for some $\mu \in \mathcal{M}$ satisfying $\mu \leq \Theta$. The condition $0 \in \mathcal{M}$ ensures that if any feasible points are known, the one with the least $f$ value is included in the pruned filter. The second condition in (7) means $\mathcal{M}$ contains an infinite sequence of marks converging to zero. Hence if $F$ contains a sequence of infeasible iterates converging to a feasible point, then $P$ retains a subsequence with the same property. There is no requirement that $\mathcal{M}$ contain members which are arbitrarily large.

A sensible choice of $\mathcal{M}$ could be, for example,

$$
\mathcal{M}=\{0\} \cup\left\{\beta^{j} \tau: \beta^{j} \tau \leq \Theta \text { and } j \in \mathbb{Z}\right\}
$$

where $\mathbb{Z}$ is the integers, $\tau>0$, and $\beta>1$. The use of a limit $\Theta$ above which points are judged purely on infeasibility means pruning marks greater than $\Theta$ are superfluous.

A template for a filter based method is stated as Algorithm 1. The first step initializes the filter. Steps 2 to 5 form a loop which processes one point in each iteration. The iterate $x^{(k)}$ is generated in step 2. The cases when $x^{(k)}$ is a worse, 
better, or comparable point to the filter $F_{k-1}$ are handled in steps 3 , 4 , and 5 respectively. Step 5 permits pruning of the filter, but does not require it. Provided $\mathcal{M}$ satisfies (7), a method conforming to this filter template will generate a sequence of iterates converging to a solution of (1) almost surely when the stopping conditions are suspended. Furthermore, with or without pruning, the sequence of filters will retain this sequence of iterates. Pruning can reduce the mark $\Theta$ above which points are judged solely on their $\theta$ values, however the sequence of $\Theta$ values must remain bounded away from zero.

Algorithm 1 The filter based template for generally constrained optimization.

1. Choose $x^{(0)} \in \Omega$. Set $F_{0}=\left\{x^{(0)}\right\}$, set $k=0$, and choose $\Theta \leq \Theta_{\max }$.

2. Increment $k$. If stopping conditions hold exit the algorithm. Otherwise choose $x^{(k)} \in \Omega$.

3. If $z \prec x^{(k)}$ for some $z \in F_{k-1}$, set $F_{k}=F_{k-1}$ and go to step 2 .

4. Set $F_{\text {temp }}=\left\{x^{(k)}\right\} \cup F_{k-1}$ and eliminate all dominated points in $F_{\text {temp. If }} x^{(k)} \prec z$ for at least one $z \in F_{k-1}$, set $F_{k}=F_{\text {temp }}$ and go to step 2 .

5. If desired, prune $F_{\text {temp }}$ to get $F_{k}$, and reduce $\Theta$ if necessary. Otherwise set $F_{k}=F_{\text {temp }}$. Go to step 2.

Pruning, as so far described, still allows the size of the filter to become arbitrarily large. However for a practical algorithm, there is a tolerance $\tau>0$ on constraint violation. Below this tolerance points are considered "feasible for all practical purposes" and such points are judged only on their objective function value. Moreover the filter has an upper limit $\Theta$ above which objective function values are ignored. For appropriate $\mathcal{M}$, these two limits yields a finite upper bound on the size of a pruned filter. Instead of defining the maximum filter size $N$ in terms of $\Theta$, we define $\Theta$ and $\mathcal{M}$ in terms of $N, \tau, \beta$, and a minimum value $J_{\min } \leq 0$ on the index $j$, as follows:

$$
\mathcal{M}=\{0\} \cup\left\{\beta^{j} \tau: \beta^{j} \tau \leq \Theta \text { and } j \in\left[J_{\min }, \infty\right) \cap \mathbb{Z}\right\} .
$$

The algorithm starts with a far larger value for $\Theta$ (such as $\Theta=\infty$ ) and reduces it as necessary to enable pruning to limit the filter size to $N$. This allows points with very large $\theta$ values to be in the filter during early iterations, which is a desirable feature. As points with low $\theta$ values are found, the limit $\Theta$ is steadily reduced to keep $|F| \leq N$, thus directing attention towards feasible parts of $\Omega$ as they are identified. The value $J_{\text {min }}$ allows points to be retained which have constraint violations less than the stopping tolerance $\tau$, which might be useful in reducing $f$. An illustration of a filter, before and after pruning, is given in Figure 2.

\subsection{Convergence}

In this and the following subsection, almost sure convergence of methods conforming to the template is shown, subject to the following assumption holding.

\section{Assumption 3}

(a) The objective function $f$ is lower semi-continuous (and hence bounded below) on $\Phi\left(\Theta_{\max }\right)$.

(b) The constraint violation $\theta$ is lower semi-continuous on $\Omega$.

(c) The set of pruning marks $\mathcal{M}$ satisfies (7). 


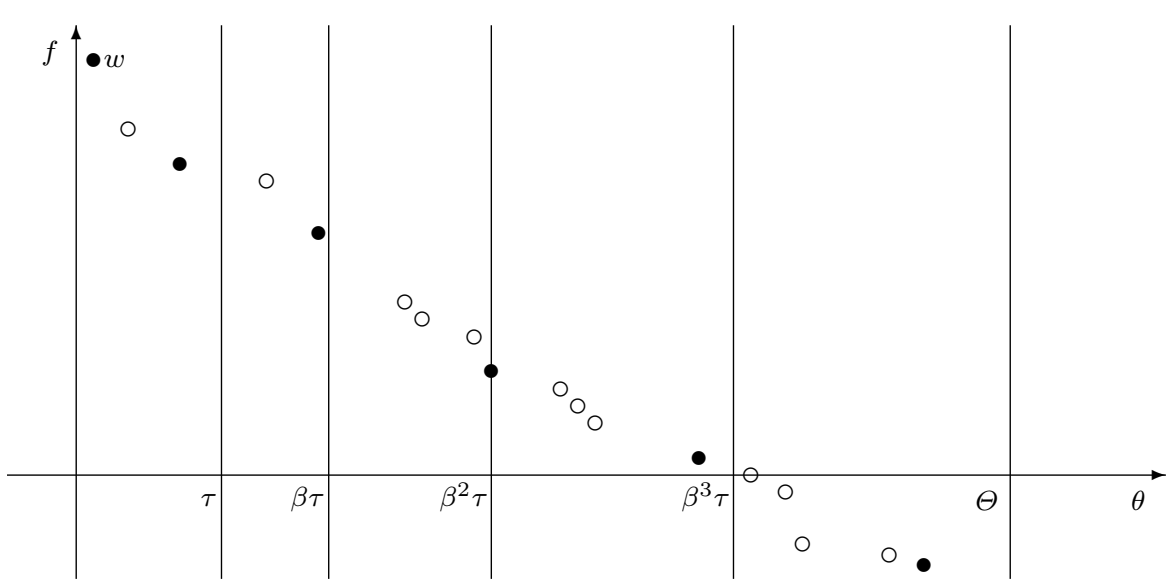

Fig. 2 This figure shows points in a filter before (circles and dots), and after pruning (dots only). The filter does not contain a feasible point. Pruning marks, as given in equation (9), are shown as vertical lines at $\mu=\beta^{j} \tau$ where $\tau$ is the tolerance on constraint violations, and $\beta$ is the scale factor between adjacent marks. Here $J_{\min }=0$ so the lowest positive mark is $\mu=\tau$. The marks do not extend beyond $\Theta$. The least infeasible infeasible point, marked $w$, is always included in the pruned filter. The remaining pruned filter points each minimize $f$ over the set of filter points with constraint violations $\theta$ less than or equal to some pruning mark.

(d) The sequence of iterates $\left\{x^{(k)}\right\}_{k=0}^{\infty}$ almost surely contains an infinite number of sample points drawn randomly from $\Omega$.

Parts (a) and (b) of this assumption are conditions on the problem. Since $\Theta$ is never increased, it suffices that part (a) holds for the initial value of $\Theta$. Parts (c) and (d) are a conditions on any algorithm conforming to the template. Condition (d) requires that the algorithm will, almost surely, look everywhere eventually. Conditions like this are very common in global optimization when confronted with black-box functions. In contrast, if global information such as Lipschitz constant is available, or interval arithmetic methods can be employed, the need to 'look everywhere' might be superfluous.

The analysis proceeds under the assumption that stopping conditions are removed and an algorithm conforming to the template is run forever. Under these conditions a variety of convergence results are shown to hold almost surely. The results in this, and the following subsection address the cases when $f_{\text {egs }}^{*}$ is infinite, and finite respectively. The first of these looks at the ability to attain feasibility from the infeasible region.

Theorem 4 Assume all iterates are infeasible, and let $w_{k}$ minimize $\theta$ over $F_{k}$ for each $k \in \mathbb{N}$. Then every cluster point $w^{*}$ of the sequence of least infeasible infeasible filter points $\left\{w_{k}\right\}$ is an essential global minimizer of $\theta$ over $\Omega$, almost surely.

Proof: The pruning strategy always retains the infeasible point with the least $\theta$ value. Hence $w_{k}$ persists in each filter until an infeasible point $w$ is found satisfying either $\theta(w)<\theta\left(w_{k}\right)$, or both $\theta(w)=\theta\left(w_{k}\right)$ and $f(w)<f\left(w_{k}\right)$. Thus $\left\{\theta\left(w_{k}\right)\right\}$ is a non-strictly decreasing sequence. This, and assumption $3(\mathrm{~d})$ imply

$$
\lim _{k \rightarrow \infty} \theta\left(w_{k}\right) \leq \inf \left\{\theta_{\text {test }} \in \mathbb{R}: m_{L}\left(\Phi\left(\theta_{\text {test }}\right)\right)>0\right\} \quad \text { almost surely. }
$$


Lower semi-continuity of $\theta$ implies any cluster point $w^{*}$ of $\left\{w_{k}\right\}$ is an essential global minimizer of $\theta$ over $\Omega$, almost surely.

This shows the template will asymptotically find a feasible point provided the measure of $\Phi(\eta)$ is positive for all $\eta>0$. This latter condition holds when, for example, the feasible region has positive measure, or a feasible point exists at which $g$ is continuous.

The next result applies directly to the sequence of best known feasible iterates, and shows that its cluster points are essential global minimizers of $f$ over $\Phi(0)$. A more general result is shown first, with this as a corollary. We define the best known point $b_{k}(\mu)$ as the point which minimizes $f$ over $F_{k} \cap \Phi(\mu)$, where $\mu \in \mathcal{M}$.

Theorem 5 Let $f$ and $\theta$ be lower semi-continuous on $\Phi(\mu)$, where $\mu \in \mathcal{M}$ satisfies $m_{L}(\Phi(\mu))>0$. Then cluster points of the sequence of best known points $\left\{b_{k}(\mu)\right\} \subset$ $\Phi(\mu)$ are essential global minimizers of $f$ over $\Phi(\mu)$, with probability one.

Proof: Let $b^{*}$ be a cluster point of $\left\{b_{k}(\mu)\right\}$ which is not an essential global minimizer of $f$ over $\Phi(\mu)$ in the topology induced on $\Omega$ by the standard topology for $\mathbb{R}^{n}$. Lower semi-continuity of $\theta$ means $\Phi(\mu)$ is closed. Hence $b^{*} \in \Phi(\mu)$. Now $\left\{x \in \Phi(\mu): f(x)<f\left(b^{*}\right)\right\}$ has positive Lebesgue measure, so this set is sampled almost surely, by Assumption 3(d). Hence a $b_{k}(\mu)$ exists satisfying $f\left(b_{k}(\mu)\right)<f\left(b^{*}\right)$, almost surely. Now $\left\{f\left(b_{k}(\mu)\right)\right\}$ is a decreasing sequence, so lower semi-continuity of $f$ means $b^{*}$ can not be a cluster point of $\left\{b_{k}(\mu)\right\}$, almost surely.

For any $\mu \in \mathcal{M}$, this result shows almost sure convergence to a point which achieves the essential global minimum $\gamma(\mu)$ over $\Phi(\mu)$. In the case when $\mu=0$ it yields the following corollary.

Corollary 6 Let $f$ be lower semi-continuous on $\Phi \equiv \Phi(0)$ and let $m_{L}(\Phi(0))>0$. Then the cluster points of the sequence of best known feasible points $\left\{b_{k}(0)\right\} \subset \Phi$ are essential global minimizers of $f$ over $\Phi$, almost surely.

Proof: The result follows directly from Theorem 5 on putting $\mu=0$.

\subsection{Convergence to an essential global solution}

Finally we establish almost sure convergence to a point in the essential global solution set $S^{*}$ for (1), when $f_{\text {egs }}^{*}$ is finite. We note that this condition implies $m_{L}(\Phi(\eta))>0$ for all $\eta>0$ via Definition 2. We define open neighbourhoods of $S^{*}$ of the form

$$
S_{\delta}^{*}=\left\{x \in \Omega: d\left(x, S^{*}\right)<\delta\right\} \text { for each } \delta>0,
$$

where $d\left(x, S^{*}\right)$ is the distance between the point $x$ and the optimal set $S^{*}$; that is to say

$$
d\left(x, S^{*}\right)=\inf \left\{\|x-s\|_{2}: s \in S^{*}\right\} .
$$

The next theorem shows that if a point is nearly feasible and nearly optimal for its level of constraint violation, then it is near to an essential global solution of (1). By generating and identifying such points an essential global solution can be located asymptotically. 
Theorem 7 Let $f_{\mathrm{egs}}^{*}$ be finite. For all sufficiently small positive $\epsilon$ and $\mu \in \mathcal{M}$,

$$
f(x)<\gamma(\mu)+\epsilon \text { and } x \in \Phi(\mu) \Rightarrow x \in S_{\delta(\epsilon, \mu)}^{*}
$$

where $\delta(\epsilon, \mu) \rightarrow 0$ as $\sqrt{\epsilon^{2}+\mu^{2}} \rightarrow 0$.

Proof: The proof is by contradiction. Assume the theorem is false, and choose monotonically decreasing sequences $\left\{\epsilon_{j}\right\}_{j=1}^{\infty}$ and $\left\{\mu_{j}\right\}_{j=1}^{\infty} \subseteq \mathcal{M}-\{0\}$, both converging to zero as $j \rightarrow \infty$. Then there exists a positive constant $\xi$ and a sequence $\left\{y_{j}\right\}_{j=1}^{\infty}$, such that

$$
y_{j} \in \Phi\left(\mu_{j}\right) \text { and } f\left(y_{j}\right)<\gamma\left(\mu_{j}\right)+\epsilon_{j} \quad \forall j \in \mathbb{N}
$$

and

$$
y_{j} \notin S_{\xi}^{*} \quad \forall j \in \mathbb{N} .
$$

The sequence $\left\{y_{j}\right\}$ lies in a compact set, and so must have cluster points. Let one such (arbitrary) cluster point be $y_{\infty}$. By replacing $\left\{y_{j}\right\}$ with a subsequence of itself if necessary, let $\left\{y_{j}\right\}$ converge to $y_{\infty}$. Now $\theta\left(y_{j}\right) \rightarrow 0$ as $j \rightarrow \infty$, and so by the lower semi-continuity of $\theta$, it follows that $\theta\left(y_{\infty}\right)=0$. Lower semi-continuity of $f$ and (10) yield the left hand inequality in

$$
f\left(y_{\infty}\right) \leq \lim _{j \rightarrow \infty} \gamma\left(\mu_{j}\right)=f_{\text {egs }}^{*}
$$

The right hand equality is via equation (6). Hence $y_{\infty} \in S^{*}$ because $y_{\infty} \in \Phi$ and $f\left(y_{\infty}\right) \leq f_{\text {egs }}^{*}$. Since $y_{j} \rightarrow y_{\infty}$ as $j \rightarrow \infty$ this contradicts (11).

This result is directly exploited by the filter method: for any fixed $\epsilon, \mu>0$ with $\mu \in \mathcal{M}$, any point $x \in \Phi(\mu)$ satisfying $f(x)<\gamma(\mu)+\epsilon$ can only be dominated or pruned by another point satisfying those conditions. Hence once the filter contains a point satisfying these conditions for a specific $\epsilon, \mu>0$ with $\mu \in \mathcal{M}$, it will always do so. Such points occur almost surely as the algorithm draws an infinite subsequence of sample points randomly from $\Omega$.

Theorem 8 Let $m_{L}(\Phi(\eta))>0$ for all $\eta>0$. Then there exists a sequence of filter points $\left\{z_{k}\right\}_{k=1}^{\infty}$ such that any cluster point $z^{*}$ of $\left\{z_{k}\right\}_{k=1}^{\infty}$ satisfies $z^{*} \in S^{*}$ almost surely, where $z_{k} \in F_{k}$ for all $k$.

Proof: Pick arbitrary positive values for $\mu_{j} \in \mathcal{M}-\{0\}$ and $\epsilon_{j}$. The definition of $\gamma\left(\mu_{j}\right)$ shows that the set

$$
T\left(\mu_{j}, \epsilon_{j}\right)=\left\{x \in \Phi\left(\mu_{j}\right): f(x) \leq \gamma\left(\mu_{j}\right)+\epsilon_{j}\right\}
$$

has strictly positive Lebesgue measure. Hence the probability that a randomly generated sample point in $\Omega$ lies in $T$ is strictly positive. The number of sample points randomly drawn from $\Omega$ is infinite with probability one, so $\left\{x^{(k)}\right\} \cap T$ is non-empty, almost surely. Let $z$ be the first such sample point generated. This point will be placed in the filter in step 4 or 5 of the template. The sample point $z$ can be removed from the pruned filter only if a new point $z_{+}$is found satisfying either $z_{+} \prec z$, or both $z_{+} \in \Phi\left(\mu_{j}\right)$ and $f\left(z_{+}\right) \leq f(z)$. Both of these possibilities imply $z_{+} \in T$.

By considering a sequence of pairs $\left(\mu_{j}, \epsilon_{j}\right)$ converging to zero as $j \rightarrow \infty$, with $\mu_{j} \in \mathcal{M}$, it is clear that the sequence of filters contains a sequence of points in $T\left(\mu_{j}, \epsilon_{j}\right)$ with $\left(\mu_{j}, \epsilon_{j}\right) \rightarrow(0,0)$, almost surely. Theorem 7 shows that this sequence converges to one or more solutions of (1), as required. 


\section{The filter OSCARS algorithm}

For convenience we refer to the filter OsCARS method as F-OSCARS, a summary of which is presented as Algorithm 2. In addition to storing $z, f(z)$, and $\theta(z)$ for each filter point $z \in F$, F-OSCARS also stores the lower and upper bounds $\ell_{k}(z)$ and $u_{k}(z)$ of $\Omega_{k}(z)$. The box $\Omega_{k}(z)$ is the region from which a random sample will be drawn if $z$ is chosen as the control point.

The basic approach of F-OSCARS is as follows: at iteration $k$ the method randomly chooses a control point $c \in F_{k-1}$. The method then draws the next iterate $x^{(k)}$ randomly from $\Omega_{k}(c)$, calculates $f$ and $\theta$ at $x^{(k)}$ and updates the filter according to the template in Algorithm 1. Step 1 in Algorithm 2 initializes the method. Steps 2 to 5 form a loop which generates and processes one sample point $x^{(k)}$ per iteration. Step 2 generates $x^{(k)}$ and inserts it into the filter. Step 2 then removes any dominated points from the filter. If $x^{(k)}$ is a better point than $F_{k-1}$ no further action needs to be taken, in which case the current iteration ends at step 3. Otherwise, steps 4 and 5 update the filter and other relevant information. Step 5 prunes the filter. When $x^{(k)}$ is a worse point to $F_{k-1}$ we have $F_{k}=F_{k-1}$ and so no pruning actually occurs, in accordance with the filter template.

In addition to the filter, the box $\Omega_{k}(c)$ must be updated at iteration $k$, where $c$ is the control point used at iteration $k$. This is done using a similar strategy to ARs. The box $\Omega_{k}(c)$ is shrunk whenever the new sample point $x^{(k)}$ is comparable to or worse than $F_{k-1}$. The box $\Omega_{k}(c)$ is shortened using the same mechanism as OSCARS. Using

$$
\Omega_{k}(c)=\left\{x \in \Omega: \ell_{k}(c) \leq x \leq u_{k}(c)\right\},
$$

the current control point $c$ remains in the filter and $\Omega_{k+1}(c) \subset \Omega_{k}(c)$ is chosen so that $c$ lies in $\Omega_{k+1}(c)$, but $x^{(k)}$ does not. This is done by moving one face of $\Omega_{k}(c)$ between $c$ and $x^{(k)}$. The face moved is orthogonal to the axis along which the component of $x^{(k)}-c$, normalized with respect to $\Omega$, is maximal. That face is moved so that it passes through the point $A c+(1-A) x^{(k)}$, where $A \in(0,1)$. Clearly $A=0$ puts the face through $x^{(k)}$, and $A=1$ through $c$. Only the control point's box is shortened; all other filter points' boxes are not changed. This completes an update of the filter and associated list of boxes $\left\{\Omega_{k}(z): z \in F\right\}$. An illustration of the cutting process is given in Figure 3.

When $x^{(k)}$ is a better point than $F_{k-1}$, all boxes in the filter (including $\Omega_{k-1}(c)$ ) remain as they are. Since $x^{(k)}$ is a better point, there exists a $z \in F_{k-1}$ for which $x^{(k)} \prec z$. However it is not necessarily true that $x^{(k)} \prec c$. Hence $\Omega_{k-1}(c)$ is not shortened; to do so would exclude a known better point $x^{(k)}$ from $\Omega_{k}(c)$. The box for the new point $x^{(k)}$ is set equal to $\Omega$.

If the box $\Omega_{k}(c)$ becomes too small, that box is reset to $\Omega$ via $\Omega_{k}(c)=\Omega$. The size $\left\|\Omega_{k}(c)\right\|_{\text {nd }}$ of $\Omega_{k}(c)$ is defined as the infinity norm of the normalized diagonal $u^{(k)}(c)-\ell^{(k)}(c)$. Specifically

$$
\left\|\Omega_{k}(c)\right\|_{\mathrm{nd}}=\max _{i=1, \ldots, n} \frac{u_{i}^{(k)}(c)-\ell_{i}^{(k)}(c)}{U_{i}-L_{i}}
$$

which is the infinity norm of the scaled main diagonal of $\Omega_{k}(c)$, where the scaling maps $\Omega$ to the unit hypercube.

The next theorem shows that F-OSCARS satisfies assumption 3(d). 


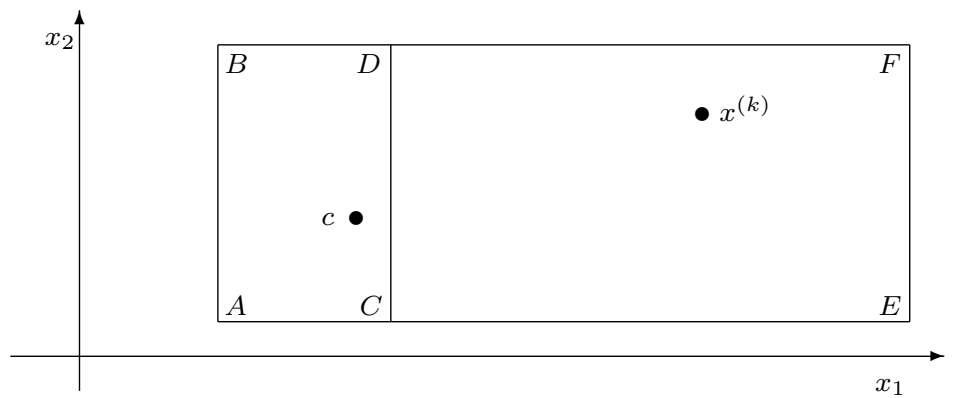

Fig. 3 The F-OSCARS cutting process: the box $\Omega_{k}(c)$ (shown as ABFE) is sampled at $x^{(k)}$. The point $x^{(k)}$ is rejected by the filter, and so $\Omega_{k}(c)$ is cut. The cut is orthogonal to the $x_{1}$ axis because $x^{(k)}-c$ has its longest normalized component along that axis. Cutting yields the new box $\Omega_{k+1}(c)$, which is ABDC.

Theorem 9 The sequence of iterates $\left\{x^{(k)}\right\}_{k=0}^{\infty}$ contains an infinite number of sample points drawn randomly from $\Omega$ with probability one.

Proof: Our approach is to show that drawing only a finite number of sample points from $\Omega$ (as opposed to those drawn from proper subsets of $\Omega$ ) is a probability zero event. Let the $K^{\text {th }}$ iteration be the last iteration at which $x^{(k)}$ is chosen randomly from $\Omega$. We partition each filter $F_{k}$ into two disjoint parts: $H_{k}$ and $E_{k}=F_{k}-H_{k}$, where $z \in H_{k}$ if and only if $\Omega_{k}(z)=\Omega$. The probability that a point is drawn randomly from $\Omega$ at iteration $k$ is $\left|H_{k}\right| /\left|F_{k}\right|$.

The only way a new point in $E_{k}-E_{k-1}$ can be found is generating a comparable or worse point in $\Omega_{k}(z) \equiv \Omega$ for some $z \in H_{k}$ and then shortening $\Omega_{k}(z)$. Once $k>K$ this is impossible. Hence $\left|E_{k}\right| \leq\left|E_{k-1}\right|$ for all $k>K$, and the probability of drawing a point randomly from $\Omega$ is at least $p=1 /\left(1+\left|E_{K}\right|\right)$ at any iteration for which $H_{k}$ is non-empty and $k>K$. Thus it suffices to show that the number of non-empty $H_{k}$ is infinite, almost surely.

Choose $T>K$ such that $H_{k}$ is empty for all $k>T$. If a better point $z$ is found at iteration $k$, this better point is included in both $F_{k}$ and $H_{k}$. Pruning does not occur because $z$ dominates another filter point, and $\left|F_{k}\right| \leq\left|F_{k-1}\right|$. Hence no better point can ever be found for all $k>T$.

All other possibilities result in the control point's box being shortened. Since $\left|E_{K}\right|$ is finite, some $z \in E_{K}$ has its box shortened an infinite number of times. To avoid $\Omega_{k}(z)$ being reset to $\Omega$ in step $5,\left\|\Omega_{k}(z)\right\|_{\text {nd }}>h_{\text {min }}$ must hold for all $k>T$. Now $\left\|\Omega_{k}\right\|_{\text {nd }} \equiv\left\|\Omega_{k}\right\|_{\infty}$ in [14] when $\Omega$ is scaled to the unit hypercube. Theorem 2 of [14] shows that shortening a box an infinite number of times without triggering a reset to $\Omega$ is a probability zero event.

Algorithm 2 The filter version of OSCARS.

1. Choose a random point $x^{(0)} \in \Omega$. Calculate $f_{c}=f\left(x^{(0)}\right)$. Set $k=m=0$, set $c=x^{(0)}$. Choose $\Theta \in\left(\tau, \Theta_{\max }\right]$ and $A \in(0,1)$. Select $h_{\min }>0$. Pick the maximum filter size $N \geq 3$. Set $F_{0}=\{c\}$ and $\Omega_{0}(c)=\Omega$.

2. Increment $k$. If stopping conditions hold exit the algorithm. Randomly select first $c \in$ $F_{k-1}$, then $x^{(k)} \in \Omega_{k-1}(c)$. Set $F_{\text {temp }}=F_{k-1} \cup\left\{x^{(k)}\right\}$ and $\Omega_{k}\left(x^{(k)}\right)=\Omega$. Remove all dominated points from $F_{\text {temp }}$, giving $F_{k}$. 
3. Set $\Omega_{k}(x)=\Omega_{k-1}(x)$ for all $x \in F_{k-1}-\{c\}$.

If $x^{(k)}$ is a better point than $F_{k-1}$, set $\Omega_{k}(c)=\Omega_{k-1}(c)$ and go to step 2 .

4. Let $i$ maximize $\left|x_{i}^{(k)}-c_{i}\right| /\left(U_{i}-L_{i}\right)$. If $c \in F_{k}$, update $\Omega_{k}(c)$ via:

(a) If $x_{i}^{(k)}<c_{i}$, set $\Omega_{k}(c)=\left\{x \in \Omega_{k-1}(c): x_{i} \geq(1-A) x_{i}^{(k)}+A c_{i}\right\}$; otherwise

(b) set $\Omega_{k}(c)=\left\{x \in \Omega_{k-1}(c): x_{i} \leq(1-A) x_{i}^{(k)}+A c_{i}\right\}$.

If $\left\|\Omega_{k}(c)\right\|_{\text {nd }} \leq h_{\text {min }}$ reset $\Omega_{k}(c)=\Omega$ and increment $m$.

5. If $\left|F_{k}\right|>N$, then prune $F_{k}$ and reduce $\Theta$ if necessary. Go to step 2 .

\subsection{Stopping conditions}

The method halted when it had satisfied two conditions. The first of these is that it had located a point at which the measure of infeasibility $\theta$ did not exceed the maximum constraint violation tolerance $\tau$. Once this first condition was satisfied the method set a mark $f_{\text {mark }}$ equal to the best known objective function value over $\Phi(\tau)$. Each time it found a point in $\Phi(\tau)$ lower than $f_{\text {mark }}-f_{\text {acc }}$ it reset $f_{\text {mark }}$ to this new lowest value. Here $f_{\text {acc }}$ is the tolerance value on changes in $f$ below which improvements in $f$ are regarded as negligible. The method halted when $K$ iterations were completed without finding a new $f_{\text {mark }}$, where $K=2 \zeta n N\left\lceil-\log _{a}\left(h_{\text {min }}\right)\right\rceil$. Here $a=1 /(1-A)$ and the ceiling function $\lceil b\rceil$ returns the smallest integer not less than $b$. This value for $K$ was chosen as follows. It takes at most $\left\lceil-\log _{a}\left(h_{\min }\right)\right\rceil$ shortenings of each face in a pair of opposite faces of a box to reduce the distance between them to less than $h_{\min }$ when $\Omega$ is scaled to the unit hypercube. Noting that all filter points collectively have at most $n N$ pairs of faces, this means each filter point will have on average at least $\zeta$ complete cycles through a range of box sizes from $\Omega$ to $h_{\text {min }}$ assuming no unfiltered points are found. Due to the stochastic nature of the method, there is no guarantee that all points will actually pass through $\zeta$ cycles.

\section{Numerical Results}

F-OSCARS was tested on 13 constrained global optimization test problems. The first 12 are from [11] and the $13^{\text {th }}$ from [12]. They appear together in [19]. These results are compared with two other methods, both of which list results for these problems. The first of these [8] is the filter based simulated annealing method FsA which employs a variant of the Nelder Mead method as a local search. Runarsson and Yao [19] present an evolutionary method (SRES) which determines the fitness of individuals in a population by a stochastic bubble sort process.

The parameter values used by our method were $\tau=10^{-6}, f_{\text {acc }}=10^{-3}, \beta=1.1$, $J_{\min }=-2, N=30, A=0.9, h_{\min }=10^{-8}, \Theta_{\max }=\infty$, and $\zeta=6$. These values give $K=2880 n$ for the stopping condition. We also used $\theta$ as defined in (3) to measure infeasibility. The squared term narrows the width of each band with $\theta>1$ during pruning, which increases the emphasis on reducing $\theta$ when $\theta$ is large.

An examination of (9) shows the largest pruning mark is bounded below by $O\left(\beta^{N} \tau\right)$. If $\beta^{N} \tau$ is very large, the pruned filter is likely to contain highly infeasible points at all times. Intensively exploring around such points can be wasteful. Numerical experimentation verifies this, with $N=60, \beta=1.5$ and $N=30, \beta=2$ both being worse than the values given just above. 
Table 1 compares results from our method (averaged over 30 runs), with those for [8] and [19]. The results for [8] and [19] have been lifted directly from those sources (Table 2 for [19]). The problems have been separated into two groups for analysis: those solved by all 3 methods, and those for which at least one method did not get an accurate estimate of the solution. For the former (g3, g4, g8, g9, g11, and g12) F-OSCARS used the fewest $f$ evaluations on all six, and sometimes significantly so. For the remaining seven problems, F-OSCARS and SRES obtained the most accurate solution on three problems, and FSA on two (FSA and F-OSCARS tied on g6). FSA and F-OSCARS each solved two more problems to within $1 \%$ of the best $f$ value known, and SREs did so on one. It is worth noting that FSA [8] has the advantage of using a version of Nelder Mead as a local search procedure, in contrast to the other two methods. F-OSCARS found points with a constraint violation less than the maximum acceptable tolerance $\tau=10^{-6}$ on all runs on all problems.

We also compare F-OSCARS with the fish swarm method P-BF AFs in [16]. The stopping rule used in [16] requires explicit knowledge of the optimal objective function value $f^{*}$ : P-BF AFS halts when either a point achieving both feasibility and optimality within tolerance is found, or the maximum number of iterations is reached. Knowledge of $f^{*}$ prevents early termination at a sub-optimal point, and avoids wasted function evaluations after optimality is achieved. F-OSCARS does not use $f^{*}$ in its stopping rule (or elsewhere), which potentially puts F-OSCARs at a disadvantage. On the harder 7 problems, P-BF AFS did better than F-OSCARS on four, worse on two, and the same on one. They tied on g6, with F-OscARs being faster on that problem. For g5 and g13 the solutions found by P-BF AFs exceeded our constraint violation tolerance of $10^{-6}$. Our method was faster on five of the six easier problems; P-BF AFS was quicker on g3.

Table 5 of [1] lists final function values for an electromagnetic-like method, a particle swarm method, and a differential evolution method for problems g1-g13. Unfortunately function counts are not listed. On four of the seven harder problems, ours obtained a better objective function value than any method listed in [1]. Not all methods in [1] were able to solve all of the six easier problems.

We compared our method to the interval methods of Sun and Johnson [20] on their example 4, which is essentially problem 4.3 of [7]. Our algorithm was able to obtain an average best function value of -4.5142 over 40 runs using an average of 24839 function evaluations per run. The better interval method of [20] obtained a best function value of -4.4828 , and required more than 100,000 function evaluations and 18,000 inclusion function evaluations to do so. The global optimum is $f^{*}=-4.5142$.

Direct [9] solved the Gomez3 problem to within $1 \%$ of the optimum after 89 function evaluations, and within $0.01 \%$ of optimum after 513 function evaluations. In contrast F-OSCARS took an average of 282 function evaluations to reach a $1 \%$ accuracy, and an average of 1369 function evaluations to reach $0.01 \%$, each averaged over 40 runs. DiRECT uses the auxiliary function $\left[f-f^{*}\right]_{+}+\sum w_{i} \max \left(g_{i}, 0\right)$ where the $w_{i}$ are positive weights and $f^{*}$ is the global minimum. DiRECT works by generating increasingly fine covers of $\Omega$, where each cover consists of hyperrectangles (or boxes) aligned with the coordinate axes. The values of $f$ and $g$ are known at the centre point of each box. At each iteration some boxes are subdivided into three equal sized boxes. DiRECT avoids having to know $f^{*}$ by subdividing all 
boxes which would be selected for some possible value of $f^{*}$. On Gomez3 DIRECT is clearly quicker.

Regis and Shoemaker [17] also solve Gomez3 to one percent accuracy in $f$, their methods taking 53 and 30 function evaluations. Whilst this is clearly impressive, their methods model the objective function using radial basis functions. They require the solution of a maximin problem over the feasible region, and minimize an approximation of the objective over the feasible region at each iteration. Radial basis function methods are highly suited to problems with computationally expensive objective or constraint functions. In contrast methods such as F-OSCARS, with its lower computational overheads, are better suited to problems for which $f$ and $g$ can be calculated cheaply. Interestingly, sub-problems of radial basis methods such as [17] have this property, and F-OSCARS could be used to solve such sub-problems.

As it stands, F-OSCARs suffers from the curse of dimensionality because it only cuts one side of a box at a time. This is a quirk of F-OSCARs, not an inherent feature of the filter approach. This curse could easily be avoided by modifying F-OSCARS so that, for example, half the faces of a box were cut at each iteration, when $n$ is large.

\section{Conclusion}

A filter based template for non-smooth black-box bound and otherwise constrained global optimization has been presented, and convergence shown almost surely under mild conditions. A variation of oscars has been implemented. This method is relatively simple, and has constant overheads per sample point. It is best suited to objective and constraint functions which are not too expensive to evaluate. Numerical results show this method is competitive in practice. There are many unexplored methods conforming to the filter template, both within the relatively new class of ARs algorithms, and elsewhere.

Acknowledgments: The authors would like to thank two anonymous referees for their comments, which have improved the paper.

\section{References}

1. M.M. Ali, C.M. Golalkhani, and J. Zhuang, A computational study of different penalty approaches for solving constrained global optimization problems with the electromagnetismlike method, Optimization 63, pp. 403-419 (2014). doi:10.1080/02331934.2012.655691

2. M.M. Ali and C. Storey, Topographical multi-level single linkage, J. Global Opt. 5, pp. 349358 (1994).

3. M.J. Appel, R. Labarre, and D. Radulović, On accelerated random search, SIAM J. Opt. 14, pp. 708-731 (2003).

4. E.G. Birgin, C.A. Floudas, and J.M. Martinez, Global minimization using an augmented Lagrangian method with variable lower-level constraints, Math. Prog. Ser. A, doi:10.1007/s10107-009-264-y

5. G. Di Pillo, S. Lucidi, and F. Rinaldi, An approach to constrained global optimization based on exact penalty functions, J Global Opt. 54, pp. 251-260 (2012). doi:10.1007/s10898-010-9582-0

6. R. Fletcher and S. Leyffer, Nonlinear programming without a penalty function, Math. Prog. ser. A 91, pp. 239-269 (2002).

7. C.A. Floudas and P.M. Pardalos, A collection of test problems for constrained global optimization problems, Lecture notes in Computer Science 455, (1990), Springer-Verlag (Berlin). 
8. A.-R. Hedar and M. Fukushima, Derivative free filter simulated annealing method for constrained continuous global optimization, J. Global Opt. 35, pp. 521-549 (2006). doi:10.1007/s10898-005-3693-z

9. D. Jones, Direct global optimization algorithm, Encyclopedia of Optimization, C.A. Floudas and P.M. Pardalos (Eds.), vol. 1, pp. 431-440 (2001), Kluwer (Dordrecht/London/Boston).

10. D. Jones, C.D. Perttunen, and B.E. Stuckman, Lipschitzian optimization without the Lipschitz constant, J. Opt. Theory Applic., 79, 157-181 (1993).

11. S. Koziel and Z. Michalewicz, Evolutionary algorithms, homomorphous mappings, and constrained parameter optimization, Evol. comput., 7, 19-44 (1999).

12. Z. Michalewicz, Genetic algorithms, numerical optimization and constraints, in Proc $6^{\text {th }}$ Int. Conf. Genetic algorithms, L. J. Eshelman, Ed., San Mateo, CA: Morgan Kaufman 1995, 151-158.

13. C.S. Pedamallu, L. Özdamar, T. Czendes, and T. Vinkó, Efficient interval partitioning for constrained global optimization, J. Global Opt., 42, 369-384 (2008). doi:10.1007/s10898008-9297-7

14. C.J. Price, M. Reale, and B.L. Robertson, One side cut accelerated random search: A direct search method for bound constrained global optimization, Optimization Letters, 8, pp. 1137-1148 (2014). doi:10.1007/s11590-013-0631-8

15. C.J. Price, M. Reale, and B.L. Robertson, A cover partitioning method for bound constrained global optimization, Opt. Methods and Software, 27, pp. 1059-1072 (2012). doi:10.1080/10556788.2011.557726

16. A.M.A.C. Rocha, M.F.P. Costa, and E.M.G.P. Fernandes, A filter-based fish swarm algorithm for constrained global optimization: theoretical and practical issues, J. Global Opt., 60, 239-263 (2014). doi:10.1007/s10898-014-0157-3

17. R.D. Regis and C.A. Shoemaker, Constrained global optimization of expensive black box functions using radial basis functions, J. Global Opt., 31, 153-171 (2005).

18. A.H.G. Rinnooy Kan and G.T. Timmer, Stochastic global optimization methods part II: multi-level methods, Math. Prog., 39, 57-78 (1987).

19. T.P. Runarsson and X. Yao, Stochastic ranking for constrained evolutionary optimization, IEEE trans. Evolutionary Computation 4, pp. 284-294 (2000).

20. M. Sun and A.W. Johnson, Interval branch and bound with local sampling for constrained global optimization, J. Global Opt., 33, pp. 61-82 (2005). doi:10.1007/s10898-004-6097-6

21. A.I.F. Vaz and L.N. Vicente, A particle swarm pattern search method for bound constrained global optimization, J. Global Opt., 39, pp. 197-219 (2007). doi:10.1007/s10898007-9133-5

22. C.-Y. Wang and D. Li, Unified theory of augmented Lagrangian methods for constrained global optimization, J. Global Opt., 44, pp. 433-458 (2009). doi:10.1007/s10898-008-93471

23. Z.Y. Wu, F.S. Bai, H.W.J. Lee, and Y.J. Yang, A filled function method for constrained global optimization, J. Global Opt., 39, pp. 495-507 (2007). doi:10.1007/s10898-007-91522

24. Z.Y. Wu, F.S. Bai, Y.J. Yang, and M. Mammadov, A new auxiliary function method for general constrained global optimization, Optimization, 62, pp. 193-210 (2013). doi: $10.1080 / 02331934.2011 .571258$ 


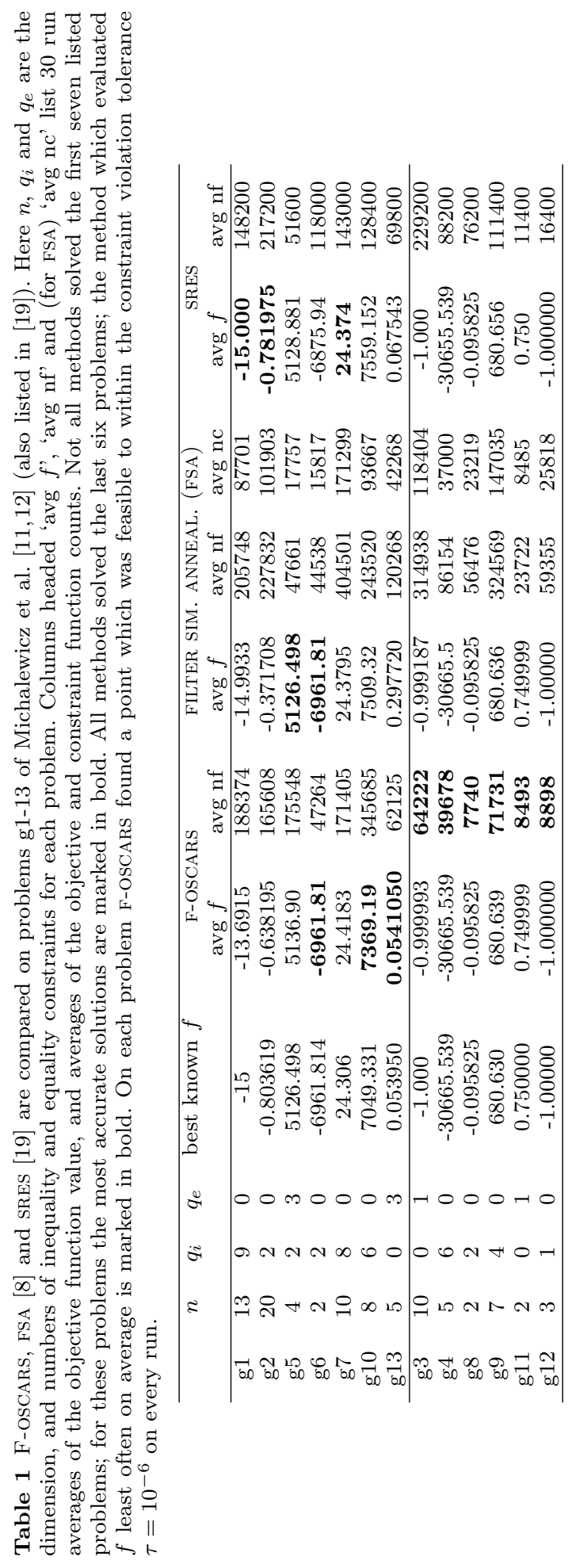

